\title{
Generation and suppression of runaway electrons in disruption mitigation experiments in TEXTOR
}

\author{
S A Bozhenkov ${ }^{1}$, M Lehnen ${ }^{1}$, K H Finken ${ }^{1}$, M W Jakubowski², \\ R C Wolf ${ }^{2}$, R Jaspers ${ }^{3}$, M Kantor ${ }^{1,3,4}$, O V Marchuk ${ }^{1}$, E Uzgel $^{1}$, \\ G Van Wassenhove ${ }^{5}$, O Zimmermann ${ }^{1}$, D Reiter ${ }^{1}$ and \\ TEXTOR team \\ ${ }^{1}$ Institute for Energy Research - Plasma Physics, Forschungzentrum Jülich GmbH, \\ EURATOM-Association, Trilateral Euregio Cluster, D-52425 Juelich, Germany \\ 2 Max-Planck-Institut für Plasmaphysik, D-17491 Greifswald, Germany \\ ${ }^{3}$ FOM Institute for Plasma Physics Rijnhuizen, Association EURATOM-FOM, \\ Trilateral Euregio Cluster PO BOX 1207, 3430 BE Nieuwegin, The Netherlands, \\ www.rijnhuizen.nl \\ ${ }^{4}$ Ioffe Institute, RAS, Saint Petersburg, 194021, Russia \\ ${ }^{5}$ Laboratoire de Physique des Plasmas/Laboratorium voor Plasmafysica, \\ LPP-ERM/KMS, Association EURATOM-Belgian State, Trilateral Euregio Cluster, \\ Brussels, Belgium \\ E-mail: s.bozhenkov@fz-juelich.de
}

\begin{abstract}
Runaway electrons represent a serious problem for the reliable operation of the future experimental tokamak ITER. Due to the multiplication factor of $\exp (50)$ in the avalanche even a few seed runaway electrons will result in a beam of high energetic electrons that is able to damage the machine. Thus suppression of runaway electrons is a task of high importance, for which reason we present here a systematic study of runaway electrons following massive gas injection in TEXTOR.

Argon injection can cause generation of runaways carrying up to $30 \%$ of the initial plasma current, while disruptions triggered by injection of helium or of mixtures of argon $(5,10,20 \%)$ with deuterium are runaway free. Disruptions caused by argon injection finally become runaway free for very large amounts of injected atoms.

The appearance/absence of runaway electrons is related to the fraction of atoms delivered to the plasma center. This so called mixing efficiency is deduced from a $0 \mathrm{D}$ model of the current quench. The estimated mixing efficiency is: $3 \%$ for argon, $15 \%$ for an argon/deuterium mixture and about $40 \%$ for helium.

A low mixing efficiency of high-Z impurities can have a strong implication for the design of the disruption mitigation system for ITER. However, a quantitative prediction requires a better understanding of the mixing mechanism.
\end{abstract}




\section{Introduction}

In ITER [1] production of runaway electrons (RE) in a disruption becomes a serious problem. Relativistic electrons accelerated in an ITER disruption are thought to be dangerous for the machine integrity [2]. A technique for suppression of runaways will be required for the reliable ITER operation. There are several such techniques under discussion: resonant magnetic perturbations, electron cyclotron heating (ECRH) or possibly lower hybrid current drive (LHCD) and massive gas injection. Resonant magnetic perturbations were demonstrated to reduce number of REs in the present day machines both in the flat-top and in disruptions. The reduction was shown in the flattop in TEXTOR [3, 4] and in disruptions in JT-60U [5] and in TEXTOR [6]. However, to predict efficiency of resonant magnetic perturbations for ITER further studies are still necessary. ECRH and LHCD are able to suppress runaway electrons in the flat-top phase owing to a related drop of the loop voltage, as found for ECRH and LHCD in FTU [7] and for LHCD in HT-7 [8]. The ECRH and LHCD suppression in disruptions is still to be proven, since the required power can be very large [9]. In this paper we discuss suppression of REs by massive gas injection (MGI) in TEXTOR. MGI has an advantage that it can also mitigate heat loads and halo currents $[10,11,12]$.

First we briefly summarize established results of the theory of runaway electrons.

Runaway electrons in tokamaks are a well known phenomenon [13, 14]. These electrons are constantly accelerated by an electric field $E$, because the drag force $\mathrm{F}$ exerted by Coloumb collisions decreases with the relative velocity $v[15]$ :

$$
\mathrm{F}=-\frac{4 \pi e^{4} n_{e} \ln \Lambda}{m_{e} v^{2}}(2+Z)
$$

In a strong electric field electrons with velocities in the domain $\mathrm{F}<e E$ run away.

In the relativistic limit the drag force $\mathrm{F}$ has a minimum $[16,17]$. Therefore runaways do not appear, if the electric field is too small:

$$
E \leq E_{C}=\frac{\min (\mathrm{F})}{e}=\frac{4 \pi e^{3} \ln \Lambda}{m c^{2}} n_{e}^{*} \approx 5.2 \cdot 10^{-22} \cdot n_{e}^{*} \mathrm{~V} / \mathrm{m},
$$

where in the last part the electron density $n_{e}^{*}$ is in $\mathrm{m}^{-3}$ and the Coulomb logarithm was estimated to be 10. Since the energy of a RE moving at $v \rightarrow c$ is much higher than the binding energy in an atom, $n_{e}^{*}$ has to include bound electrons: $n_{e}^{*}=n_{e}+n_{\text {bound }}$.

If $E>E_{C}$, the number of runaways grows due to two mechanisms: (i) primary or Dreicer mechanism; and (ii) secondary or avalanche mechanism.

In the Dreicer mechanism the number of REs increases due to diffusion of electrons in the momentum space from the thermal into the runaway region. The generation rate $S_{D}$ for plasmas changing on a timescale longer than that of the flux formation and for $E_{D}>E$ was determined in a series of papers $[18,19,20,21]$ :

$$
\begin{aligned}
S_{D} & \approx 0.35 \cdot n_{e} \nu_{e 0}\left(\frac{E_{D}}{E}\right)^{3 \zeta / 8} \exp \left(-\frac{E_{D}}{4 E}-\sqrt{2 \zeta}\left(\frac{E_{D}}{E}\right)^{1 / 2}\right) \approx \\
& \approx 3.82 \cdot \ln \Lambda \frac{\left(n_{e}\left[\mathrm{~cm}^{-3}\right]\right)^{2}}{\left(T_{e}[\mathrm{eV}]\right)^{3 / 2}}\left(\frac{E_{D}}{E}\right)^{3 \zeta / 8} \exp (\cdots)\left[\mathrm{m}^{-3} \mathrm{~s}^{-1}\right]
\end{aligned}
$$


where $\zeta=(Z+1) / 2, \nu_{e 0}$ is the collisional frequency at the thermal velocity and the Dreicer field $E_{D}$ is the field necessary to make thermal electrons become runaways:

$$
E_{D}=\frac{4 \pi e^{3} n \ln \Lambda}{T_{e}} \approx 2.61 \cdot 10^{-11} \cdot \ln \Lambda \frac{n_{e}\left[\mathrm{~cm}^{-3}\right]}{T_{e}[\mathrm{eV}]}\left[\mathrm{V} \cdot \mathrm{m}^{-1}\right]
$$

The primary generation rate falls off as the electron density $n_{e}$ gets larger. The dependence given by equation 3 was confirmed experimentally [22].

It is to be noted that the Dreicer field $E_{D}$ is sometimes called critical. However, we use the term critical field in the sense given by equation 2 .

The secondary mechanism is driven by collisions between runaway and thermal electrons. If in such a collision the energy gained by a thermal electron is enough for the electron to leap into the runaway domain, an avalanche with the generation rate $S_{A}=n_{R E} \gamma_{R E}$ develops $[23,24]$. The growth rate $\gamma_{R E}$ is $[25,26]$ :

$$
\begin{aligned}
\gamma_{R E} & =\frac{e\left(E-E_{C}\right)}{m_{e} c \ln \Lambda} \sqrt{\frac{\pi \phi}{3(Z+5)}} \cdot \\
& \cdot\left(1-\frac{E_{C}}{E}+\frac{4 \pi(Z+1)^{2}}{3 \phi(Z+5)\left(E^{2} / E_{C}^{2}+4 / \phi^{2}-1\right)}\right)^{-1 / 2} \approx \\
& \approx 59.975 \cdot\left(E-E_{C}\right) \sqrt{\frac{\phi}{Z+5}}(\cdots)^{-1 / 2}\left[\mathrm{~s}^{-1}\right]
\end{aligned}
$$

where $\phi \approx\left(1+1.46 \sqrt{A_{R E}}+1.72 A_{R E}\right)^{-1}$ is a function of the inverse aspect ratio $A_{R E}=a_{R E} / R_{0}$ and in the last part the electric fields are in $\mathrm{V} \cdot \mathrm{m}^{-1}$. Experimentally the secondary mechanism was first detected in TEXTOR [22]. Later it was confirmed in a similar way in HT-7 [27] and was found to be necessary for the explanation of the REs in JET disruptions [28, 29].

Avalanching of REs leads to a qualitative difference for a disruption in plasmas with large plasma currents. The number of e-foldings $\alpha$ during the current quench equals to $[30]$ :

$$
\alpha \sim \frac{I}{I_{A} \ln \Lambda} \sim 50
$$

where $I_{A} \approx 0.02 \mathrm{MA}$ is the Alfvén current and the estimation is given for the $15 \mathrm{MA}$ inductive ITER scenario [1]. Because of the multiplication by $\exp (50)$ even a few seed runaways produced, for example, by Compton scattering will result in a runaway beam carrying up to $70 \%$ of the pre-disruptive current [2]. Since an abrupt loss of such a beam can damage the machine, it is necessary to prevent generation of runaways.

A condition for full suppression of REs in a disruption is given by equation 2, where the electric field $E$ is the field induced during the current decay. The requirement can be met by increasing $n_{e}^{*}$ by massive injection of impurity atoms. Physically the suppression takes place because of the growth of the drag force due to a larger number of the field electrons.

Use of high-Z impurities seems to be advantageous due to the inclusion of bound 


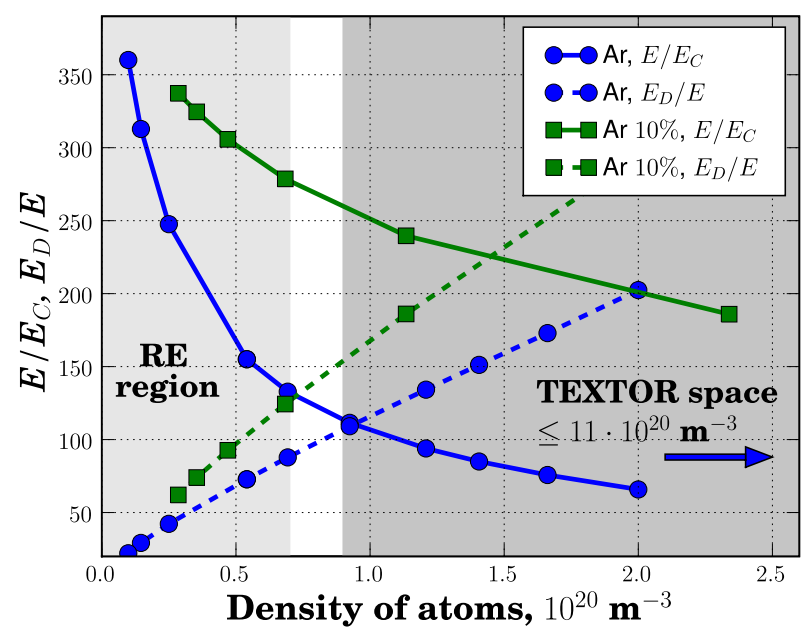

Figure 1. Dependence of the ratios $E / E_{C}(-)$ and $E_{D} / E(---)$ on density. Shown are two cases: (i) argon and (ii) mixture of $10 \%$ of argon with $90 \%$ of deuterium. The legend is given in the figure. "RE region" is the region where the RE generation in TEXTOR is to be expected; "TEXTOR space" is the estimation of argon density for the TEXTOR MGI experiments.

electrons (equation 2). A possible point of confusion is that high-Z ions cool the plasma and intensify the induced field $E$, as obvious from Ohm's law:

$$
E=\eta \cdot j \approx 5 \cdot 10^{-5} \cdot \frac{Z_{e f f} \ln \Lambda}{T_{e}^{3 / 2}} \cdot j
$$

The problem must be considered self consistently: it is necessary to find the variation of the ratios $E / E_{C}$ and $E_{D} / E$ with the density of introduced impurities $n_{\text {imp }}$. For plasma parameters relevant to the tokamak disruptions, i.e. for $T_{e}$ below the maximum of the radiative cooling rate $L_{i m p}$, the gain in the critical field $E_{C}$ turns out to outweigh the growth of $E$. Figure 1 illustrates the dependence of the ratio $E / E_{C}$ on the density of introduced impurities $n_{i m p}$ for TEXTOR parameters for two cases: (i) argon (•) and (ii) mixture of $10 \%$ argon with $90 \%$ of deuterium (ם). The ratio was calculated from equations 2, 7 and from the power balance:

$$
\eta j^{2}=n_{i m p}^{2} \cdot Z_{e} L_{i m p}\left(T_{e}\right) \Rightarrow n_{i m p}^{2}=f\left(T_{e}\right) \cdot j^{2},
$$

where $Z_{e}$ is an average charge state and $f$ is a function only of electron temperature. The atomic data for argon are taken from from [31, 32]. As the number of atoms is increased the ratio reduces and tends to unity. The difference between the two cases arises from a larger number of bound electrons in the case of pure argon. These calculations give the values only at the very beginning of the current quench. A full self consistent evolution of the runaway population and of the plasma parameters will be considered later in this paper. We also note that the calculations presented here are similar to those presented in [10] on basis of the KPRAD model.

In figure 1 one also finds the dependence of the parameter $E_{D} / E$ on density. This ratio 
characterizes the magnitude of the Dreicer mechanism (equation 3). Its importance will be discussed in section 3 .

Coronal equilibrium used in estimating the ratios $E / E_{C}$ and $E_{D} / E$ is not valid for densities and temperatures required for the suppression of REs in ITER: $n_{e}$ up to $10^{17}$ $\mathrm{cm}^{-3}, T_{e}<5 \mathrm{eV}$. Therefore resolving the boundary $E / E_{C}=1$ calls for a collisionalradiative model, possibly with opacity being taken into account [33]. For the densities reached in TEXTOR $\left(n_{e} \leq 2 \cdot 10^{14} \mathrm{~cm}^{-3}\right)$ the coronal limit is still sufficient [31, 32].

Previously a systematic study of REs after gas injection was performed in JT-60U $[34,35]$ by using fueling valves (injection time $\sim 100 \mathrm{~ms}$, flow rates $\sim 5 \cdot 10^{21}$ atoms $/ \mathrm{s}$ ). Injections of argon, krypton or xenon were found to provoke RE generation, while by simultaneous injection of one of these gases with deuterium through two separate valves it was possible to avoid runaways. The drawback of this study is that the slow puffing, whose timescales are too slow for a real time mitigation, was used.

In experiments with fast gas injection (injection time $\leq 10 \mathrm{~ms}$, flow rates $\geq 10^{25}$ atoms/s) RE generation has not been investigated thoroughly. Tore Supra shows, that puffs of helium ( $<10^{23}$ atoms) suppress REs in the disruptions where the RE generation would otherwise occur [36]. DIII-D reports on a weak runaway population $I_{R E}<0.1 \cdot I_{p}$ for helium, neon and argon $[37,38]$. In experiments in ASDEX Upgrade REs are rare and appear only in discharges with densities below $4 \cdot 10^{19} \mathrm{~m}^{-3}$ and $B_{t}>2 \mathrm{~T}$. Runaway electrons were not specifically addressed in ASDEX Upgrade [12]. The absence of REs in the experiments listed above can be explained by a low toroidal field $B_{t}$, i.e. close to the RE threshold of $2 \mathrm{~T}$ [39], together with a small secondary multiplication. The achievement of the condition $E / E_{C}<1$ has not been demonstrated yet.

In TEXTOR a fast gas injection system with parameters similar to the systems at Tore Supra, DIII-D and ASDEX Upgrade is used. Timescales of the system are about a few milliseconds only and make it suitable for disruption mitigation. The number of injected atoms divided by the plasma volume, i.e. the density of impurities, is by an order of magnitude larger than in JT-60U. Moreover, the TEXTOR system allows a variation of the number of particles puffed before the thermal quench.

We show that argon injection can be followed by the REs carrying up to $30 \%$ of the initial plasma current, while disruptions triggered by injection of helium or of mixtures of argon $(5,10,20 \%)$ with deuterium are runaway free. Disruptions caused by argon injection become runaway free for large amounts of injected atoms. These results can be explained by a change of the primary generation with the impurity density. The full suppression of runaway electrons, i.e. $E / E_{C}<1$, is not achieved.

The appearance/absence of runaway electrons is related to the fraction of atoms delivered to the plasma centre. This so called mixing efficiency is deduced from a 0D model of the current quench and turns out to be very low. A low mixing efficiency of high-Z impurities can have a strong implication for the design of the disruption mitigation system for ITER.

The article is organized in the following way: in section 2 the experimental setup is introduced; section 3 describes the experimental results; the model of the current quench 


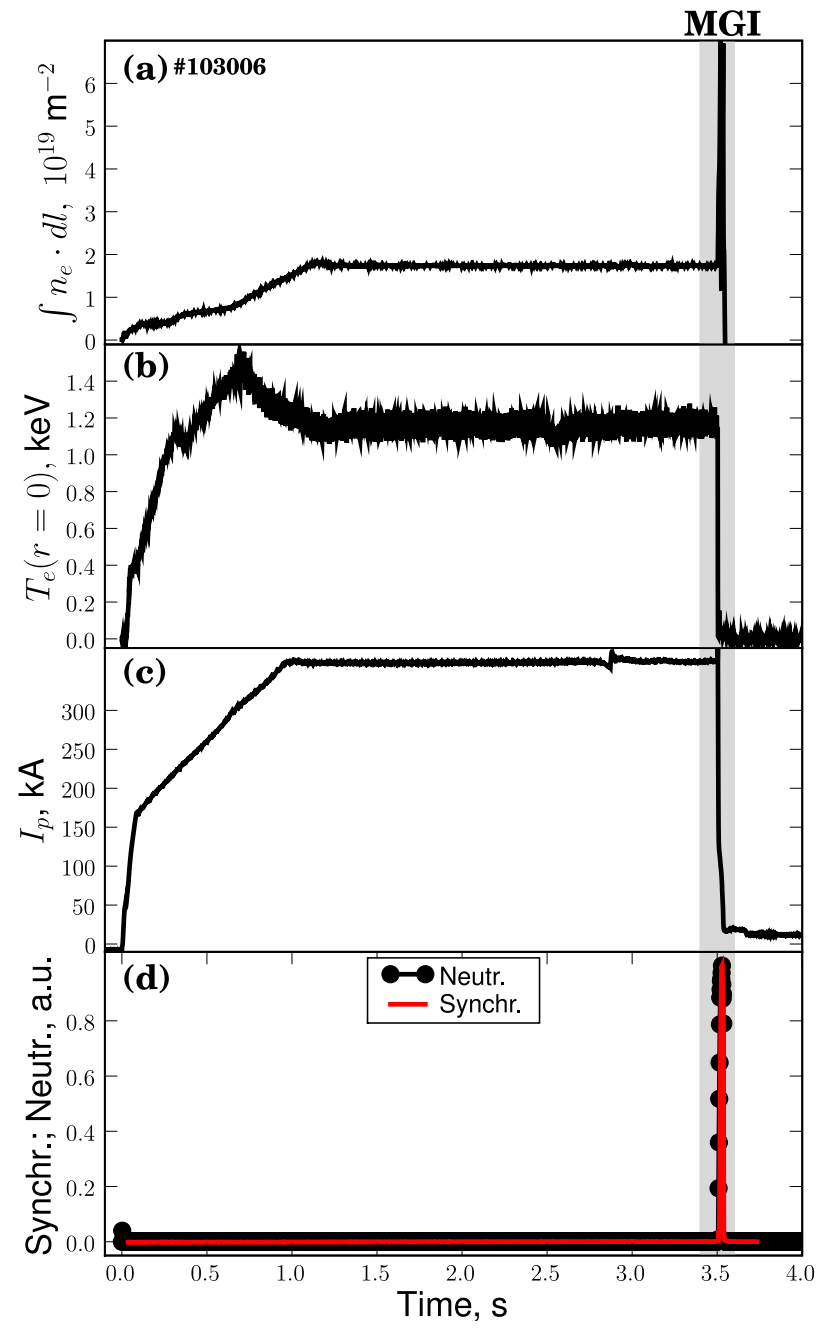

Figure 2. Time traces of the main plasma parameters in the MGI experiments. (a) - electron density integrated along a chord passing through the plasma centre. (b) central electron temperature. (c) - plasma current. (d) - runaway signals: neutron flux and synchrotron emission. A time moment of the MGI experiment is highlighted. The discharge was terminated by injection of $5.3 \cdot 10^{21}$ atoms of argon.

is developed and applied in section 4; and conclusions are drawn in section 5.

\section{Description of the experiment}

The experiments were conducted in the limiter tokamak TEXTOR. Identical ohmic discharges were terminated by gas injection during the flat-top phase. Typical time traces of main plasma parameters during these experiments are presented in figure 2. Shown are line integrated electron density, central electron temperature, plasma current, synchrotron emission and neutron flux. Before MGI (a moment of the MGI is highligthed in the figure) plasma is stable and no runaway electrons are observed. The disruption initiated by the injection develops on a millisecond timescale. In the current quench of 
the disruption a strong runaway generation takes place, see figures $2 \mathrm{~d}$ and 3 . Generation and suppression of runaway electrons were studied by varying the type and amount of the injected gas.

The main parameters of the used discharges and of the machine are: major radius $R_{0}=1.75 \mathrm{~m}$, minor radius $a=0.46 \mathrm{~m}$, toroidal field on the axis $B_{t}=2.25 \mathrm{~T}$, plasma current $I_{p}=350 \mathrm{kA}$, line averaged electron density $<n_{e}>=2 \cdot 10^{19} \mathrm{~m}^{-3}$, electron content of the discharge $n_{\text {tot }}=1.1 \cdot 10^{20}$, plasma volume $V_{p}=7.3 \mathrm{~m}^{-3}$ and volume of the vacuum vessel $V \approx 17 \mathrm{~m}^{-3}$. The chosen toroidal magnetic field was above the well known threshold of about $2 \mathrm{~T}$ for the generation of REs in disruptions [39], which was also confirmed in TEXTOR.

The gas injections were performed by means of the fast valve described in [42, 43]. Owing to the absence of any ferromagnetic materials in the valve construction it is especially suitable for operation close to the machine. At TEXTOR the valve was installed at a distance of about $1.3 \mathrm{~m}$ away from the plasma edge: $0.8 \mathrm{~m}$ of a tube with an inner diameter of $40 \mathrm{~mm}$ and $0.5 \mathrm{~m}$ of free flow.

The number of injected atoms was varied in the range $(1.4-20) \cdot 10^{21}$, which corresponded to 14 - 200 times the electron content of the discharge, by changing the working gas pressure from 1.5 to 20 bar. The atoms were injected in a typical time of $0.5-5.1$ ms (the e-folding time of gas pressure), with the exact number depending on the gas species and valve configuration. It is worth mentioning that the upper limit on the number of particles was imposed solely by the TEXTOR vacuum system.

The used gases were: $\mathrm{D}_{2}$, He, Ar and the mixtures of Ar with $\mathrm{D}_{2}$ containing 5,10 or $20 \%$ of argon. Argon was chosen as a balance between high-Z and the reaction time, while deuterium and helium represented the case of low-Z. The mixtures of argon with deuterium were addressed in an attempt to shorten the reaction time and to increase the delivery rate of argon at the expense of the number of electrons.

Runaway electrons in the current quench phase become evident as a long lasting current plateau: the plasma current deviates from the exponential decay and preserves a constant value for tens of milliseconds. The population of high energy runaway electrons was also monitored by recording their synchrotron emission with an IR camera. The 14-bit camera SBF-125 (InSb, $320 \times 256$ pixels) is located in the equatorial plane and observes plasma tangentially in the electron approach direction. The view covers central part of the plasma with approximate dimensions of $30 \times 30 \mathrm{~cm}$. The registered spectral range is $\lambda=3-5 \mu \mathrm{m}$, which makes this diagnostics sensitive to runaway electrons with energies above $20 \mathrm{MeV}[40,41]$. The system can record full images at a frequency of up to $394 \mathrm{~Hz}$ and reduced images at a frequency of up to $13 \mathrm{kHz}$. In the experiments presented here the camera ran at a frame rate of about $2 \mathrm{kHz}$.

Additionally, losses of the runaway electrons were monitored with uncollimated neutron detectors. Neutrons are produced in the nuclear photoeffect by photons from the bremsstrahlung of runaway electrons on ions of the wall. Consequently a strong neutron flux is an evidence for lost runaway electrons with energies above about $10 \mathrm{MeV}$. The detectors employed at TEXTOR are of liquid scintillator type and are also sensitive to 


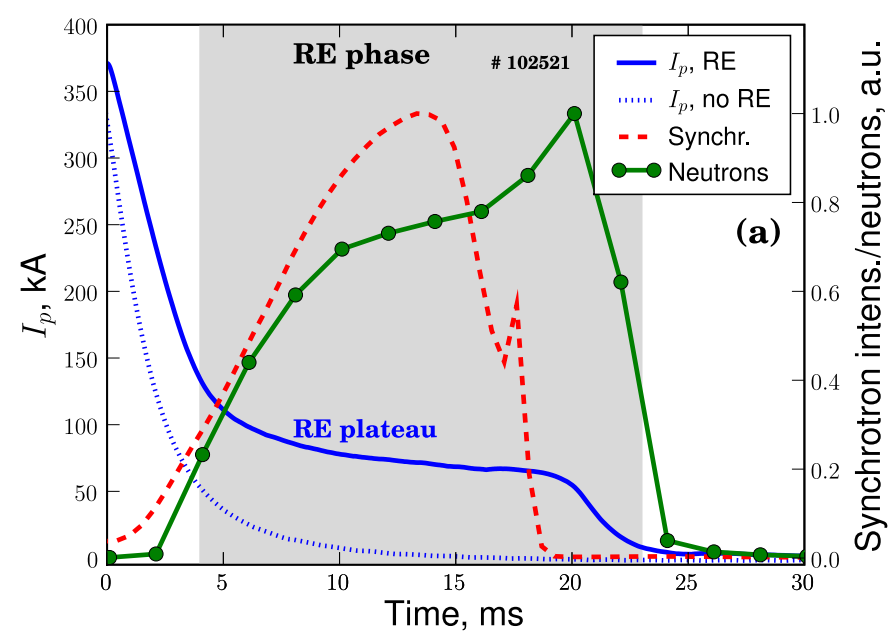

(b)

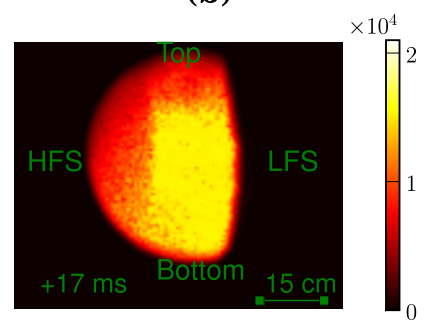

Figure 3. Runaway electrons in the argon experiments. (a) Time traces of several signals for the discharge terminated by $5.3 \cdot 10^{21}$ atoms of argon. Also an example of the current quench without runaway electrons after injection of $20.9 \cdot 10^{21}$ atoms of argon is given. (b) an example of the 2D image of runaway beam obtained by the IR camera, the same shot. The frame was taken at the maximum of the synchrotron emission: $t=+17 \mathrm{~ms}$.

hard $\gamma$ emission. The $\gamma$ emission was to some extent attenuated by a lead shielding. The disadvantage of the neutron detectors is their proneness to saturation at high count rates. The saturation was monitored by comparing two detectors with different sensitivities, of which only one will be shown below. In this work only a relative variation of the signals is discussed. Having been obtained for an uniform toroidal distribution of the neutron source, the absolute calibration of the detectors could not be applied for runaway discharges.

Qualitative dynamics of the disruptions was visualized with an ultra-fast framing camera PSI-5 viewing the plasma tangentially with a spatial resolution of about $1.5 \mathrm{~cm}$ and frame rates of up to $500 \mathrm{kHz}$. The camera was equipped with an interference filter selecting ionization stages of the injected elements.

Besides, the following TEXTOR diagnostics were used for the analysis: ECE temperature measurements, density measurements with the HCN interferometer, temperature and density measurements with Thomson scattering with repetition rate of $5 \mathrm{kHz}$.

\section{Experimental results}

In the MGI experiments with argon it was found that if the amount of injected atoms was less than $(14 \pm 2) \cdot 10^{21}$, strong RE generation was provoked. At the same time, runaway electrons were absent in disruptions caused by injection of $\mathrm{D}_{2}$, He and mixtures of $\mathrm{Ar}$ with $\mathrm{D}_{2}$ in the whole investigated range $(1.4-20) \cdot 10^{21}$ atoms. In this section the 


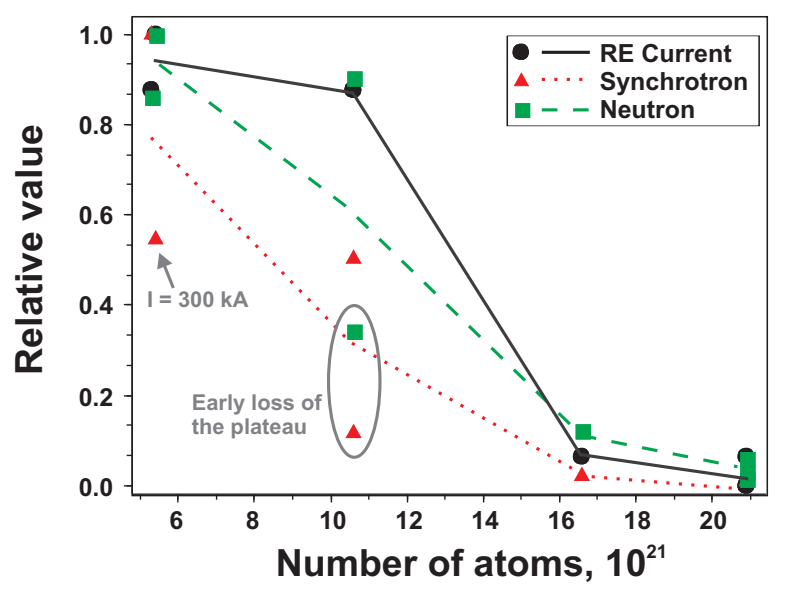

Figure 4. Suppression of runaway electrons by large amounts of argon. The point marked as $I=300 \mathrm{kA}$ corresponds to the shot with the plasma current of $I=300 \mathrm{kA}$. The points marked as "early loss of the plateau" represent the shots where the REs were terminated earlier than usually.

main experimental results are presented.

After triggering the valve four stages can be distinguished in the experiment [48]: (i) the propagation of gas through the vacuum delivery tube; (ii) cooling of the plasma edge predisruptive phase; (iii) rapid loss of the plasma thermal energy - thermal quench; and (iv) resistive current decay - current quench. A detailed analysis of this phenomenology and a discussion of the mechanism of the disruption will be given elsewhere. Here we consider the generation of runaway electrons in the current quench, which starts $2-6$ $\mathrm{ms}$ after the valve trigger. In the following analysis, the time is given relative to the beginning of the current decay, i.e. relative to the moment when the current reaches its maximum in the transient current peak.

The appearance of runaway electrons following an injection of $5.3 \cdot 10^{21}$ atoms of argon is illustrated in figure $3(\mathrm{a})$. The current $(-)$ considerably deviates from the exponential decay at $5 \mathrm{~ms}$ and a runaway plateau is formed. The plateau lasts about $15 \mathrm{~ms}$ until it is finally terminated by the fast loss of the beam to the wall. The dynamics of the runaway beam and the processes leading to the RE termination are not considered in the present work.

A significant population of high energy runaways is evidenced by their synchrotron emission and by the neutron $/ \gamma$ flux (-- and $\bullet$ correspondingly). The synchrotron intensity presented in figure 3(a) is an average over an entire camera frame. An example of the $2 \mathrm{D}$ image of the runaway beam obtained by the IR camera is given in figure 3(b).

The synchrotron intensity starts to grow at the beginning of the current quench and reaches its maximum after $10-15 \mathrm{~ms}$. The drop of the intensity takes place by about $2 \mathrm{~ms}$ earlier than the loss of the runaway plateau occurs, which is explained by a shift of the runaway beam outward of the field of view and by a jitter in the timing of the IR camera. The temporal behaviour of the neutron $/ \gamma$ flux resembles that of the 
synchrotron emission. But the falloff of the neutron flux coincides with the loss of the current plateau within the time resolution of the detectors of $2 \mathrm{~ms}$. Since the neutrons are produced mainly by REs striking the wall, the integral of the signal represents the total number of REs accelerated to the appropriate energies (see figure 4).

As the number of injected argon atoms is increased beyond about $(14 \pm 2) \cdot 10^{21}$ the plateau disappears. An example of the current quenching without formation of the runaway plateau after an argon injection is also shown in figure $3($ a) $(\cdots \cdots)$. In this case the current decays in an exponential way.

The results concerning generation and suppression of runaway electrons after the argon puffs are summarized in figure 4, where the magnitude of the runaway current plateau $(\bullet)$, the peak intensity of the synchrotron emission $(\boldsymbol{\Delta})$ and the integral of the neutron flux (ם) are plotted in dependence on the number of argon atoms. To improve statistics an additional shot with slightly lower plasma current of $300 \mathrm{kA}$ is presented. It has to be noted that for low number of argon atoms at least one of the neutron detectors was saturated.

Both, the low and high energy $(\varepsilon>10 \mathrm{MeV})$ runaway electrons are suppressed if the number of the atoms exceeds about $(14 \pm 2) \cdot 10^{21}$. Runaway current, synchrotron emission and number of neutron events are reduced by an order of magnitude.

The RE suppression observed experimentally is due to the suppression of the Dreicer mechanism only. The condition $E / E_{C}<1$ is not achieved. This will be shown later in the modeling section, where the power balance is taken into account.

To understand reasons for the primary generation, it is worthwhile returning to figure 1 , where one finds the dependence of the parameter $E_{D} / E$ on argon density. This ratio determines the magnitude of the primary generation mechanism being the dominant one in TEXTOR. The larger $E_{D} / E$ is, the less runaways are generated (equation 3 ).

Since the dependence of the primary generation rate $S_{D}$ on $E_{D} / E$ is mainly exponential, it is easy to identify the parameter space, where the RE generation with the runaway plateau above $10 \mathrm{kA}$ is to be expected. For a typical TEXTOR discharge with the plasma current of $350 \mathrm{kA}$ this region lies below $0.6 \cdot 10^{14} \mathrm{~cm}^{-3}$, with the avalanche multiplication of 5 having been taken into account. The region is marked as "RE region" in figure 1 . In the same figure the density to be expected for an uniform distribution of the injected argon in the TEXTOR experiments is also highlighted ("TEXTOR space").

In spite of being outside of the "RE region", the TEXTOR experiments do show considerable generation of runaway electrons. Consequently only a part of the injected atoms is mixed into the plasma core and suppresses the RE generation. Comparing the experimental suppression "threshold" of $n_{\text {imp }} \geq 5 \cdot 10^{14} \mathrm{~cm}^{-3}$ with the theoretical one results in an efficiency of mixing of about $10 \%$. In the next section, by using a 0D model of the current quench we refine this value to be $3 \%$.

Such an imperfect mixing is consistent with the phenomenology of the discharge termination by massive gas injection. Gas is introduced at the plasma edge and does not penetrate deeply prior to the disruption. No density increase in the plasma centre is observed before the thermal quench (figures 5 and 6 ). The thermal quench is too short 

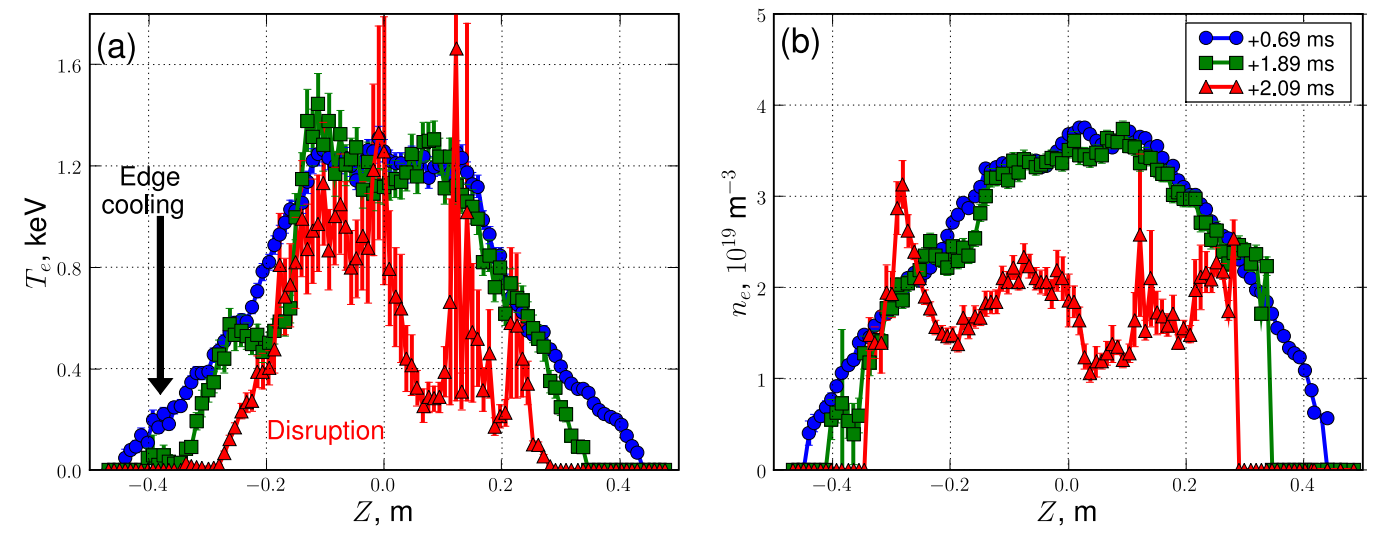

Figure 5. Evolution of the temperature (a) and density (b) profiles in the MGI experiments. Shown are the profiles taken before the gas puff $(\bullet)$, just before the disruption ( $\mathbf{\square})$ and at the disruption onset $(\boldsymbol{\Lambda})$. The discharge was terminated by mixture of $10 \%$ of argon with deuterium. The Z-coordinate is counted from the midplane along the laser beam. The laser beam passes vertically, $9 \mathrm{~cm}$ away from the geometrical centre of the tokamak vessel. The time is given relative to the valve trigger. Errorbars are given in the figure.

to provide complete mixing. The cooling of the plasma inside $q=2$ lasts less than 0.5 $\mathrm{ms}$ in the case of argon and argon/deuterium mixtures, while the time for argon from the plasma edge to reach the centre at the sound velocity is about $1.5 \mathrm{~ms}$.

In figure 5 one finds the electron temperature and density profiles measured with Thomson scattering. Shown are the profiles taken before the gas puff, just before the disruption and at the onset of the disruption for a discharge terminated by mixture of argon with deuterium. Before the disruption the temperature and density in the plasma centre are not disturbed. Only the edge region outside approximately $r=30 \mathrm{~cm}(q \approx 2)$ is cooled by the injected gas ("Edge cooling" in figure 5). It was not possible to extend these measurements to the post-disruptive phase because of low electron temperatures and an overwhelming radiation of the impurities.

The shallow penetration of impurities prior to the thermal quench is also confirmed by observations of impurity radiation in the visible range (figure 6 ). The images were obtained with the aid of the tangentially viewing camera in Ar II light $(\lambda \approx 611 \mathrm{~nm})$. The time resolution of these measurements was $40 \mu \mathrm{s}$. Several auxiliary elements are indicated in the figure to explain the view: the window of the viewing port - dark blue line; TEXTOR inner wall (high field side) - light blue points; centre of the discharge green point; radius of $30 \mathrm{~cm}(q \approx 2)$ - green circle. The frames were chosen to be as close as possible to the times of the temperature and density profiles presented in figure 5 , with the exception of the first one. Before the thermal quench the emission front propagates from the very edge $(r \geq 46 \mathrm{~cm})$ towards the centre, but does not reach it. At the disruption onset the emission is located outside of the $q \approx 2$ flux surface.

The evolution of density, temperature and emission profiles in the experiments with 


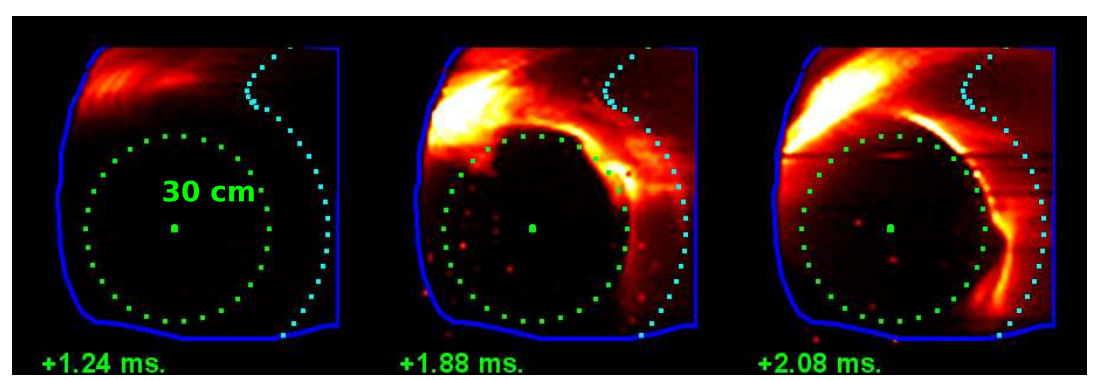

Figure 6. Emission of the injected impurities before the thermal quench. The shown discharge is the same as in figure 5. The camera was equipped with the Ar II interference filter $(\lambda \approx 611 \mathrm{~nm})$ and provided the time resolution of $40 \mu \mathrm{s}$. The window is highlighted with the dark blue line. The light blue points denote the TEXTOR inner wall (high field side). The green point marks the discharge centre. The green circle shows $r=30 \mathrm{~cm}(q \approx 2)$. The time is given relative to the valve trigger.

other gases is identical to the one described above.

Another effect to be discussed here is the possible generation of runaway electrons by the "hot-tail" or "burst" mechanism $[49,50]$. This mechanism was brought up to explain bursts of runaway generation observed $1 \mathrm{~ms}$ after the $T_{e}$ drop in DIII-D experiments on the plasma shutdown by "killer" pellets [51]. The reason for these bursts is the difference in cooling times for electrons in the tail of the electron energy distribution and for those in the thermal bulk. If the cooling time of the plasma is short enough, then the electrons in the tail are not thermalized and represent a seed population of runaway electrons. In the experiments presented here no evidences of an early generation of runaway electrons like in the DIII-D experiments were observed. Even if such runaway electrons appeared, we would expect them to be lost in the transient current peak before the current decay. The current peak is related to the global rearrangement of the current density profile and to large MHD activity and is not reported to be present in "killer" pellet experiments. Moreover, the cooling times in the argon experiments are comparable to those in the experiments with mixtures of argon with deuterium. Nevertheless, in the last case runaways are not observed even for half the number of injected atoms. Therefore we would not expect this generation mechanism to be of importance for the TEXTOR experiments.

The unimportance of the "hot-tail" mechanism for the TEXTOR experiments is partially confirmed by an observation that the runaway electrons generated in the flattop of the discharge are lost before the current quench, figure 7. This discharge differs from the standard discharges used in the rest of the work: the generation of runaways during the current ramp-up and the flat-top was intentionally provoked by a high loop voltage and a low electron density. REs are detected through their synchrotron radiation (figure 7b), which grows up to the disruption. Figures $7 \mathrm{c}$ and $7 \mathrm{~d}$ are scaled to show the loss during the disruption. Just before the current quench the synchrotron radiation drops to the background level of the thermal radiation of the walls. This observation hints that the REs observed in the disruptions are generated in the current quench. 

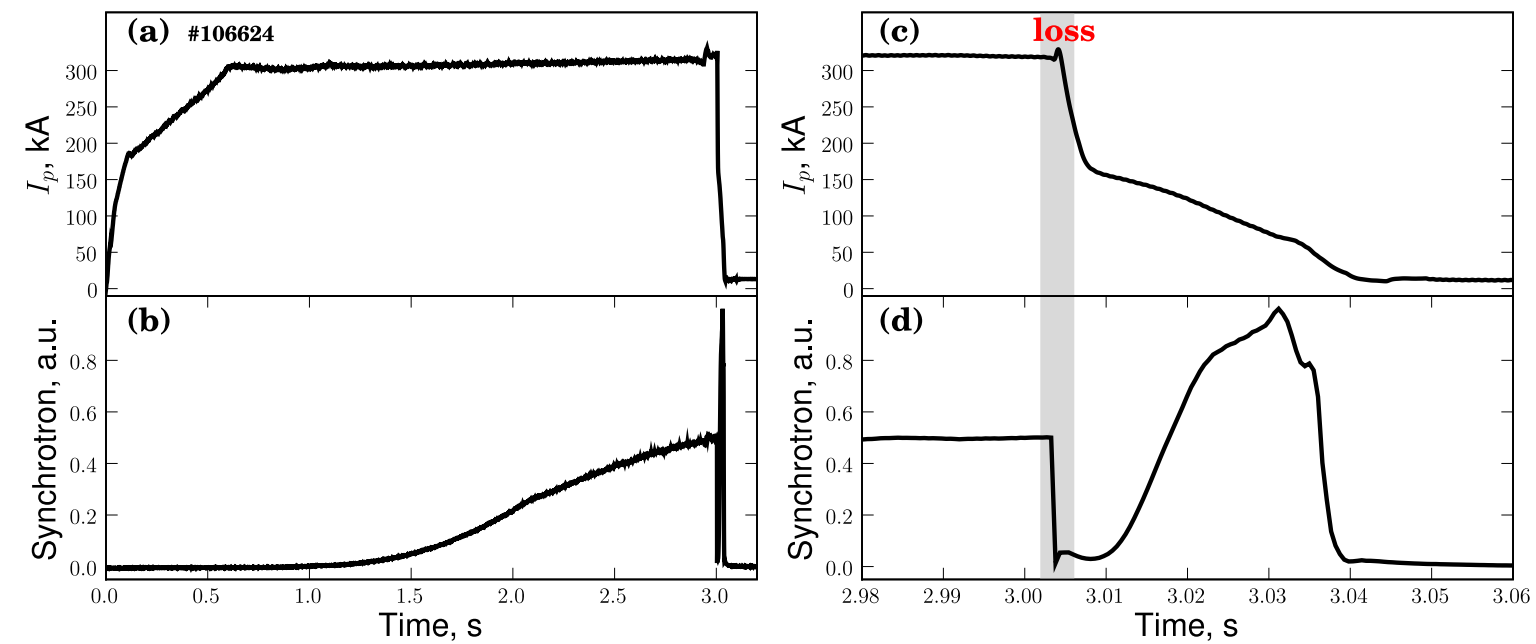

Figure 7. Loss of runaway electrons generated in the flat-top phase of the discharge during the disruption. (a) - plasma current. (b) - synchrotron radiation. (c) and (d) are the same as (a) and (b) but are scaled to show the loss before the current quench.

\section{Model of the current quench}

In this section a zero dimensional model of the current quench including the generation of REs is applied to fit the experimental results. The main outcome of the modelling is the volume average density of impurity atoms.

The description of the current evolution and the power balance in the model is standard and similar to, for example, the KPRAD model used to analyse DIII-D experiments [37]. However, the model presented here also takes into account the primary and secondary $\mathrm{RE}$ generation mechanisms and the replacement of the Ohmic current by the RE one. A similar treatment of the RE generation was previously given in [28, 45] and in [29]. In the last case the energy of REs was also calculated self-consistently. The omission of the energy evolution is not restrictive, as the typical time for an electron to be accelerated to $v \approx c$ is much shorter than the timescale in question. The listed RE models do not include the power balance.

It is to be noted that there exist several more sophisticated models dealing with runaway electrons like the $1 \mathrm{~d}$ codes described in $[46,47]$ or the kinetic approach in the codes ARENA [14] and CQL3D [26, 49]. In spite of being simpler than other available codes, the model used here is adequate for the description of the current quench. Since the plasma parameters in postdisruptive plasmas are poorly known, such a model provides a good order of magnitude estimation with only one essentially free variable. While the application of a more elaborated model like an $1 \mathrm{~d}$ code would require the knowledge of the entire density and current profiles. 
Turning to the model, the current quench is described by four coupled equations: an equation for the generation of runaways $n_{R E}$, an equation for the plasma current $I_{\Omega}$, an equation for the current induced in the vessel $I_{v}$ and the power balance:

$$
\begin{aligned}
& \frac{d n_{R E}}{d t}=f_{\text {prim }}+\left(\gamma_{R E}-\gamma_{\text {loss }}\right) n_{R E} \\
& \frac{d}{d t}\left(L I_{\Omega}+L_{R E} I_{R E}+L_{v} I_{v}\right)=-2 \pi R_{0} E \\
& \frac{d}{d t}\left(I_{v}+I_{\Omega}+I_{R E}\right)=-\frac{I_{v}}{\tau_{v}} \\
& \eta \frac{I^{2}}{S^{2}}=n_{e} n_{i m p} L_{i m p}
\end{aligned}
$$

$S_{D}$ and $\gamma_{R E}$ were introduced in equations 3 and 5 . Due to the current profile flattening in the transient current peak, the inductances $L, L_{R E}$ are equal to $\mu_{0} R_{0} \cdot\left(\ln \left(\frac{8 R_{0}}{r}\right)-\frac{7}{4}\right)$, with $R_{0}$ being the tokamak major radius and $r$ being the plasma or runaway beam minor radius. The mutual inductance between the plasma and the vessel is taken to be equal to the vessel self-inductance $L_{v} \approx 2.9 \mu \mathrm{H}$. The resistive time of the vessel $\tau_{v}$ equals 1.45 ms. The current $I_{\Omega}$ and the induced field $E$ are related by equation 7 . The RE current is estimated as $I_{R E}=e c n_{R E} S_{R E}$. Coronal equilibrium is assumed in calculating the electron density $n_{e}=n_{D}+Z \cdot n_{i m p}$ and the radiative cooling rate $L_{i m p}$. The atomic data are taken from [31, 32] for argon and from [44] for helium. The deuterium density $n_{D} \approx 1.2 \cdot 10^{13} \mathrm{~cm}^{-3}$ is estimated for an uniform distribution of the initial plasma particle content. The initial conditions are: $n_{R E}(0)=0, I_{\Omega}(0)=I_{p}, I_{v}(0)=0$.

The plasma radius is assumed to decrease linearly in time according to the experimental findings pictured in figure 8 . The example represents an experiment with argon/deuterium mixture recorded in Ar II light $(\lambda \approx 611 \mathrm{~nm})$. During the current quench the radius decreases from about 0.4 to about $0.25 \mathrm{~m}$ owing to a nonuniform distribution of the impurities.

The minor radius of the runaway beam is estimated from 2D IR images.

Free parameters of the model are the initial temperature $T_{e}$ or equivalently the density $n_{i m p}$ and the loss rate of runaway electrons $\gamma_{l o s s}$. These parameters were chosen in such a way as to provide the best fit to the experimental current time trace. The main out-

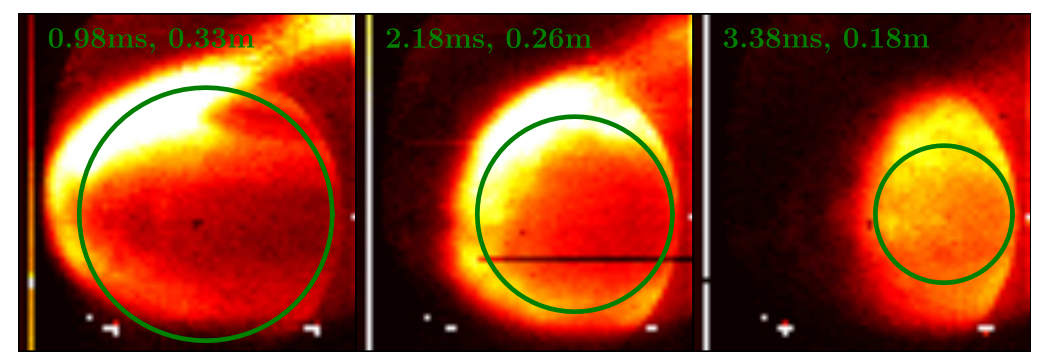

Figure 8. Plasma channel contraction during the current quench. The discharge was terminated by injection of $12.7 \cdot 10^{21}$ atoms of mixture Ar $10 \%+\mathrm{D}_{2} 90 \%$. The observations were made in ArII light $(\lambda \approx 611 \mathrm{~nm})$. 

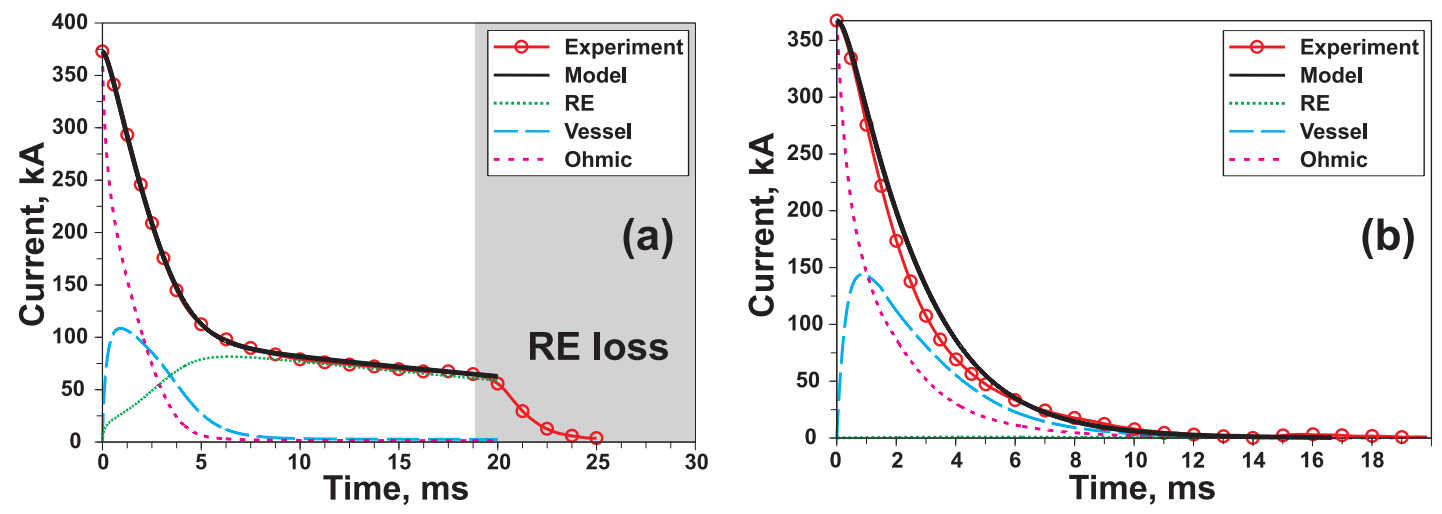

Figure 9. Modeling of the current quench. (a) - injection of $5.3 \cdot 10^{21}$ atoms of argon. Parameters of the model: $\gamma_{\text {loss }}=50 \mathrm{~s}^{-1}, n_{\text {atoms }}=0.77 \cdot 10^{13} \mathrm{~cm}^{-3}$. (b) injection of $20.9 \cdot 10^{21}$ atoms of argon. Parameters of the model: $\gamma_{\text {loss }}=200 \mathrm{~s}^{-1}$, $n_{\text {atoms }}=2.71 \cdot 10^{13} \mathrm{~cm}^{-3}$. Note that the circles "Experiment" do not show the time resolution of measurements, but are given to facilitate the comparison with the model.

come of such parametric fitting is the average density of impurities $n_{i m p}$, which does not depend strongly on the assumed loss rate $\gamma_{\text {loss }}$.

In figure 9 (a) the result of the fitting is shown for a puff of $5.3 \cdot 10^{21}$ atoms of argon. Plotted are the Ohmic current (- - - ), the current carried by runaway electrons $(\cdots \cdots)$, the current excited in the vessel $(---)$, the total modelled current $(-)$ and the current measured experimentally $(O)$. The parameters used for this simulation are given in the caption to the figure. The total modelled current coincides with the experimental one until the runaway plateau is lost. After approximately $5 \mathrm{~ms}$ the whole current is carried by the runaway electrons. It is worth mentioning that the loss rate of REs resulting from the model resembles the behaviour of the neutron fluxes.

A reasonable agreement between the modelled and measured currents can be achieved also in the cases without runaway plateau, as it is demonstrated in figure 9(b) for a discharge terminated by injection of $20.9 \cdot 10^{21}$ atoms of argon. In this case the current carried by runaway electrons is negligible and the evolution is determined by the resistive decay and the power balance.

If the REs are present, it is interesting to analyze which mechanism is responsible for their generation. A comparison of the primary ( - ) and secondary (- - - ) generation rates is given in figure 10 . The curves are normalized to the maximum of the primary mechanism. The Dreicer mechanism prevails for a short time at the very beginning when the electric field is relatively high. After less than half a millisecond the primary generation is overtaken by the RE exponentiation. Though staying lower in magnitude than the peak Dreicer generation the secondary mechanism acts ten times longer contributing to the RE density. The RE density $(\ldots \cdots)$ is shown in the same figure, this curve is normalized to its own maximum. From the point where the avalanching starts to dominate the RE density grows by a factor of 5 . This is in a good agreement with the simple estimate from equation 6 . It is seen that despite affecting the RE density, the 


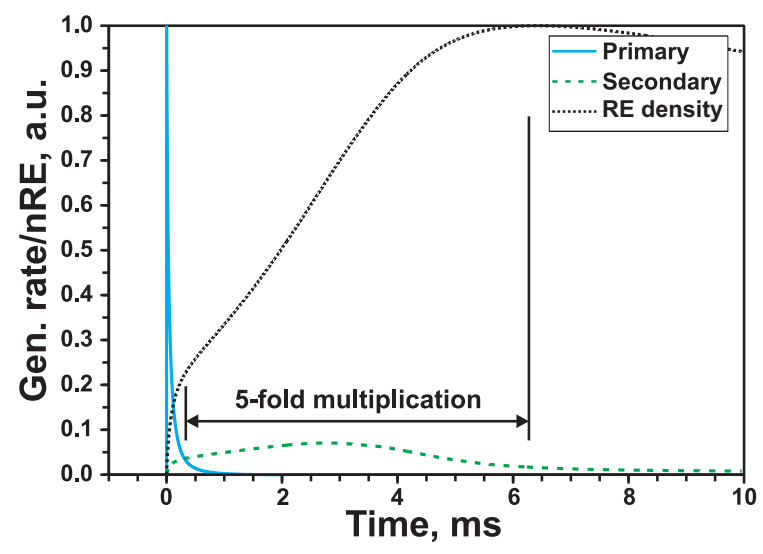

Figure 10. Comparison of the generation mechanisms. The primary and secondary generation rates are normalized to the peak value of the primary mechanism. The RE density ("population") is normalized to its own maximum.

secondary mechanism is, nevertheless, weak. If the primary mechanism is suppressed, the runaway electrons are absent in TEXTOR disruptions.

The impurity densities found by fitting are compiled in figure 11 in dependence on the density to be expected for an uniform distribution of the injected atoms: $n_{\exp }=f \cdot N / V$. Where $f$ is the fraction of atoms injected before the disruption. The argon densities $n_{\text {imp }}$ scale linearly with the expected one. But the absolute value of the ratio $n_{\text {imp }} / n_{\text {exp }}$, which can be labeled as mixing efficiency, is only about $3 \%$. It is such a low mixing efficiency that is responsible for the appearance of runaway electrons in the TEXTOR experiments with argon. If all atoms were delivered to the plasma centre, then even the smallest amount of argon used in the experiments would prevent RE generation. This fact agrees with the rough estimation from figure 1.

A similar modeling can be performed for the experiments with helium and with argon/deuterium mixture, if in the last case the preservation of the mixture composition is implied. The mixing efficiency of Ar/D mixture turns out to be of the order of $15 \%$. A higher number of the delivered free electrons prevents the formation of REs. Injection of helium results in the mixing efficiency of the order of $40 \%$. However, because of the constant current decay rate in the experiments with helium there is no clear linear dependence in this case. The main reason for this is likely to be the low radiative cooling of helium, which is by three orders of magnitude lower than that of argon and of intrinsic impurities. In contrast to the other experiments, even a carbon concentration of $1 \%$ in the initial plasma would affect the power balance considerably. Therefore, the helium result should be considered as preliminary giving only the order of magnitude.

Additionally, the modelling reveals that $E / E_{C}$ stays above 1 in all TEXTOR experiments including the puffs of helium $\left(E / E_{C} \approx 30\right)$ and mixtures of argon $\left(E / E_{C} \approx 200\right)$. $\mathrm{RE}$ generation is possible and is determined by the seed population resulting from the primary mechanism and by the competing loss channels. If the secondary multiplication factor were comparable to ITER (equation 6), a small seed RE population would be 


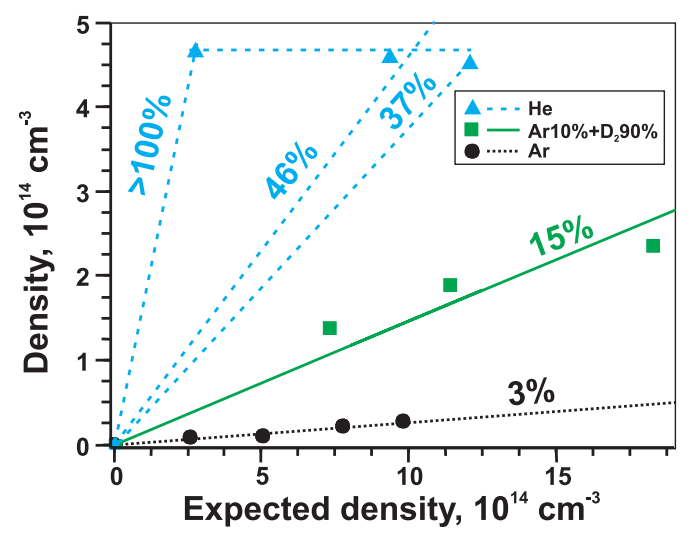

Figure 11. Mixing efficiency. Mixing efficiency is defined as the ratio of the density $n_{i m p}$ found from the model to that expected for an uniform distribution of the injected impurities.

enormously enhanced. Nevertheless, a favourable tendency of lowering the ratio $E / E_{C}$ by increasing the gas amount is observed.

It is still necessary to discuss the accuracy of the determined density $n_{i m p}$. To assess the error bars a sensitivity analysis was performed. All essential parameters of the model were varied: the resistive time constant of the vessel by a factor of 1.5 ; the vessel selfinductance by a factor of 1.5; the initial plasma radius from 0.4 to $0.3 \mathrm{~m}$; the radius of the runaway beam in the range $0.05-0.2 \mathrm{~m}$; the cooling rate $L_{i m p}$ by a factor of 2 ; the primary generation rate $S_{D}$ by a factor of 10; the secondary generation rate $\gamma_{R E}$ by a factor of 2 ; the initial conditions on the currents $\pm 30 \%$. Basing on this analysis we derive the uncertainty to be about a factor of 2 , which turns out to be mainly determined by the variation of the initial plasma radius, i.e. by the poorly known initial current density.

\section{Conclusions}

The systematic investigation of the generation of runaway electrons in the current quench after massive gas injection has been presented. Disruptions triggered by argon injection were found to be prone to runaway generation, even though the number of atoms was at least by a factor of 30 larger than the plasma electron content. Only in the experiments with the number of atoms exceeding about $(14 \pm 2) \cdot 10^{21}$, which corresponds to $140 \pm 20$ times the electron content, the runaway population was negligible. Suppression of runaway electrons by a larger number of atoms was confirmed experimentally. It is worth mentioning that runaway electrons are absent in natural TEXTOR disruptions, so that the effect of "small" argon injections can be opposite to the one expected. On the other hand, injections of similar amounts of deuterium, helium and of mixtures of argon with deuterium (5, 10, 20\% of argon) turned out to be runaway free. 
We exclude the "burst" or "hot-tail" mechanism from the consideration basing on the following experimental facts: (i) runaway electrons are not observed in the experiments with argon deuterium mixtures, even though the cooling times are almost the same as in the argon case; and (ii) we expect any fast electrons to be lost in the transient current peak, which is partially confirmed by the loss of the runaway electrons generated in the flat-top before the current quench.

A simple zero dimensional model of the current quench was developed and applied to fit the experimental data. The model included an equation for the generation of runaway electrons, an equation for the plasma current, an equation for the current induced in the vessel and the power balance. The major free parameter of the model was the density of impurity ions. By varying this density a reasonable agreement with experiment could be achieved.

Modelling led us to conclude that the major reason for the appearance of runaway electrons in the argon experiments is poor mixing of the delivered atoms into the plasma core. The mixing efficiency, i.e. the ratio of the number of atoms delivered to the core to the number of atoms injected prior to the disruption, is only $3 \%$ for argon, $15 \%$ for a mixture of argon with deuterium and $40 \%$ for helium, with the inaccuracy being about a factor of 2. However, in the case of helium the number is very preliminary due to a large influence of intrinsic impurities. We provisionally note an obvious decrease of mixing efficiency with $\mathrm{Z}$.

The model also reveals, that the absence of runaway electrons in TEXTOR is only due to the suppression of the primary (Dreicer) generation mechanism and because of low secondary multiplication about a factor of 5 . The desired condition for the full suppression $E / E_{C}<1$ was not achieved.

The imperfect mixing is consistent with the phenomenology of the shutdown. Gas is injected from the plasma edge and the disruption is initiated well before deep gas penetration. No density increase in the plasma centre is observed before the thermal quench. The thermal quench is too short to provide complete mixing of atoms. A poor mixing of injected atoms into the plasma has been recently confirmed by direct density measurements with the $\mathrm{CO}_{2}$ interferometer at ASDEX Upgrade. The rise of the density averaged along a central chord after the thermal quench in ASDEX Upgrade is about a factor of $1.5-2$ only [12].

The mixing efficiency was determined for the volume averaged density: a toroidal symmetry was assumed. Toroidal symmetry can be violated, but up to now no measurements of a degree of this violation exist. This question has to be addressed in future experiments, especially in view of its importance for the localized radiative heat loads on the first wall.

A low mixing efficiency of high- $Z$ impurities can have a critical impact on the design of the disruption mitigation system for ITER. Mixing of less than $5 \%$ of argon atoms de facto degrades the advantage of having large number of bound electrons. Assuming even a very conservative field of $50 \mathrm{~V} \cdot \mathrm{m}^{-1}$ during the current quench, one finds the impurity density required for runaway suppression to be at least $10^{17} \mathrm{~cm}^{-3}$ even for argon. The 
resulting pressure rise in the vessel is about $5 \mathrm{mbar}$, which can be critical for the vacuum system and for some diagnostics. However, a quantitative prediction requires a better understanding of the mixing mechanism.

It is still necessary to understand reasons of poor mixing and to verify its scalings with plasma parameters and with $Z$. These questions as well as the correct determination of the density of impurity atoms for suppression of runaway electrons on basis of collisionalradiative model are of high importance.

In addition, we would like to note, that, because of the good reproducibility, the runaway generation after argon injections can be conveniently used to study the physics of the runaway electrons in disruptions. Among interesting questions are the following: confinement of runaway electrons after disruption, parameter scalings, heat loads produced by the losses of runaway electrons, application of the alternative suppression techniques like resonant magnetic perturbations.

\section{References}

[1] Shimada M et al 2007 Progress in the ITER Physics Basis, Chapter 1: Overview and Summary Nucl. Fusion 47 S1-17

[2] Hender T C et al 2007 Progress in the ITER Physics Basis, Chapter 3: MHD stability, operational limits and disruptions Nucl. Fusion 47 S128-202

[3] Finken K H, Abdulaev S S, Jakubowski M W, Jaspers R, Lehnen M and Zimmermann O 2006 Losses of runaway electrons during ergodization Nucl. Fusion 46 S139-S144

[4] Finekn K H, Abdulaev S S, Jakubowski M W, Jaspers R, Lehnen M, Schlickeiser R, Spatschek K H, Wingen A and Wolf R 2007 Runaway losses in ergodized plasmas Nucl. Fusion 47 91-102

[5] Yoshino R and Tokuda S 2000 Runaway electrons in magnetic turbulence and runaway current termination in tokamak discharges Nucl. Fusion 40 1293-1309

[6] Lehnen M, Bozhenkov S A, Abdulaev S S and Jakubowski M W 2008 Suppression of Runaway Electrons by Resonant Magnetic Perturbations in TEXTOR Disruptions Phys. Rev. Lett. 100 $255003(4 \mathrm{pp})$

[7] Martin-Solis J R, Esposito B, Sanchez R, Granucci G and Panaccione L 2005 Comparison of runaway dynamics in LH and ECRH heated discharges in the Frascati Tokamak Upgrade Nucl. Fusion 45 1524-1533

[8] Chen Z Y et al 2006 Dynamics of runaway electrons in lower hybrid current drive plasmas in the HT-7 tokamak Plasma Phys. Control. Fusion 48 1489-1499

[9] Esposito B et al 2005 Mitigation of disruption-generated runaways by means of ECRH 32nd EPS Conference on Plasma Phys. Tarragona, 27 June - 1 July 2005 ECA Vol.29C, P-5.072

[10] Whyte D G et al 2003 Disruption mitigation with high-pressure noble gas injection Journal of Nuclear Materials 313-316 1239-46

[11] Granetz R, Whyte D G, Izzo V A, Biewer T, Reinke M L, Terry J, Bader A, Bakhtiari M, Jernigan T and Wurden G 2006 Gas jet disruption mitigation studies on Alcator C-Mod Nuclear Fusion 46 1001-1008

[12] Pautasso G et al 2008 Plasma shut-down with impurity puff on ASDEX Upgrade Nucl. Fusion 47 900-13

[13] Knoepfel H and Spong D A 1979 Runaway electrons in toroidal discharges Nucl. Fusion 19 785-829

[14] Helander P, Eriksson L-G and Andersson F 2002 Runaway acceleration during magnetic reconnection in tokamaks Plasma Phys. Control. Fusion 44 B247-B262 
[15] Trubnikov B A 1965 Reviews of Plasma Physics vol 1 ed Leontovich M A (New York: Consultants Bureau) p 105

[16] Connor J W and Hastie R J 1975 Relativistic limitations on runaway electrons Nucl. Fusion 15 $415-24$

[17] Fussmann G 1979 On the motion of runaway electrons in momentum space Nucl. Fusion 19 327-34

[18] Gurevich A V 1961 On the theory of runaway electrons Sov. Phys.-JETP 12 904-12

[19] Lebedev A N 1965 Contribution to the theory of runaway electrons Sov. Phys.-JETP 21 931-3

[20] Kulsrud R M, Sun Y-C, Winsor N K and Fallon H A 1973 Runaway electrons in a plasma Phys. Rev. Lett. 31 690-693

[21] Cohen R H 1976 Runaway electrons in an impure plasma Phys. Fluids 19 239-44

[22] Jaspers R, Finken K H, Mank G, Hoenen F, Boedo J A, Lopes Cardozo N J and Schüller F C 1993 Experimental investigation of runaway electron generation in TEXTOR Nucl. Fusion 33 $1775-1785$

[23] Sokolov Yu A, "Multiplication" of accelerated electrons in a tokamak JETP Lett. 29 218-21

[24] Jayakumar R, Flesischmann H H and Zweben S J 1993 Collisional avalanche exponentiation of runaway electrons in electrified plasmas Phys. Lett. A 172 447-51

[25] Rosenbluth M N and Putvinski S V 1997 Theory for avalanche of runaway electrons in tokamaks Nucl. Fusion 37 1355-1362

[26] Chiu S C, Rosenbluth M N, Harvey R W and Chan V S 1998 Fokker-Planck simulations mylb of knock-on electron runaway avalanche and bursts in tokamaks Nucl. Fusion 38 1711-1721

[27] Chen Z Y, Wan B N, Lin S Y, Shi Y J, Hu L Q, Gong X Z, Lin H and Asif M 2006 Measurement of the runaway electrons in the HT-7 tokamak Rev. Sci. Instrum 77013502 (5 pp)

[28] Gill R D, Alper B, de Baar M, Hender T C, Johnson M F, Riccardo V and contributors to the EFDA-JET Workprogramme 2002 Behaviour of disruption generated runaways in JET Nucl. Fusion 42 1039-44

[29] Plyusnin V V, Riccardo V, Jaspers R, Alper B, Kiptily V G, Mlynar J, Popovichev S, de La Luna E, Andersson F and JET EFDA contributorsa 2006 Study of runaway electron generation during major disruptions in JET Nucl. Fusion 46 277-84

[30] Putvinski S, Barabaschi P, Fujisawa N, Putvinskaya N, Rosenbluth M N ans Wesley J 1997 Halo current, runaway electrons and disruption mitigation in ITER Plasma Phys. Control. Fusion 39 B157-71

[31] Fournier K B, Cohen M, May M J and Goldstein W H 1998 Ionization State Distribution and Radiative Cooling Rate for Argon in a Low-density Plasma ADNDT 70 231-54

[32] Fournier K B, Cohen M, May M J and Goldstein W H 2000 Erratum:Ionization State Distribution and Radiative Cooling Rate for Argon in a Low-density Plasma ADNDT 74 333-4

[33] Lukash V E, Mineev A B and Morozov D Kh. 2007 Influence of plasma opacity on current decay after disruptions in tokamaks Nucl. Fusion 47 1476-84

[34] Bakhtiari M, Kawano Y, Tamai H, Miura Y, Yoshino R and Nishida Y 2002 Fast plasma shutdown scenarios in the JT-60U tokamak using intense mixed gas puffing Nucl. Fusion 42 1197-204

[35] Bakhtiari M et al 2005 Study of plasma termination using high-Z noble gas puffing in the JT-60U tokamak Nucl. Fusion 45 318-25

[36] Martin G, Sourd F, Saint-Laurent F, Bucalossi J and Eriksson L G 2005 Disruption mitigation on TORE Supra 32nd EPS Plasma Conference (Tarragona 2005)

[37] Whyte D G et al 2002 Mitigation of tokamak disruptions using high-pressure gas injection Phys. Rev. Lett. 89055001

[38] Hollmann E et al 2005 Measurements of impurity and heat dynamics during noble gas jet-initiated fast plasma shutdown for disruption mitigation in DIII-D Nucl. Fusion 45 1046-55

[39] Riccardo V and JET EFDA contributors 2003 Disruption and disruption mitigation Plasma Phys. Control. Fusion 45 A269-A284

[40] Finken K H, Watkins J G, Rusbüldt D, Corbett W J, Dippel K H, Goebel D M and Moyer R A 1990 Observation of infrared synchrotron radiation from tokamak runaway electrons in 
TEXTOR Nucl. Fusion 30 859-870

[41] Jaspers R, Lopes Cardozo N J, Donne A J H, Widdershoven H L M and Finken K H 2001 A synchrotron radiation diagnostic to observe relativistic runaway electrons in a tokamak plasma Rev. Sci. Instrum. 72 466-470

[42] Savtchkov A, Finken K H and Mank G 2002 Development of a fast valve for mitigating disruptions in tokamaks Rev. Sci. Instrum. $\mathbf{7 3} 3490-3$

[43] Bozhenkov S A, Finken K H, Lehnen M and Wolf R C 2007 Main characteristics of the fast disruption mitigation valve Rev. Sci. Instrum. $\mathbf{7 8} 033503$

[44] Post D E, Jensen R V 1977 Steady-State Radiative Cooling Rates for Low-Density, HighTemperature Plasmas ADNDT 20 397-439

[45] Jaspers R, Lopes Cordozo N J, Schüller F C, Finken K H, Grewe T and Mank G 1996 Disruption generated runaway electrons in TEXTOR and ITER Nucl. Fusion 36 367-73

[46] Eriksson L-G, Helander P, Andersson F, Anderson D and Lisak M 2004 Current dynamics during disruptions in large tokamaks Phys. Rev. Lett. 92205004

[47] Smith H, Gál K, Fehér T, Fülöp T and Helander P 2007 Runaway electron generation during plasma shutdown by pellet injection 34th EPS Conference on Plasma Physics (Warsaw 2007)

[48] Bozhenkov S A, Finken K H, Lehnen M and Wolf R C 33rd EPS on Plasma Physics, Rome 2006, P-2.162

[49] Harvey R W, Chan V S, Chiu S C, Evans T E, Rosenbluth M N and Whyte D F 2000 Runaway electron production in DIII-D killer pellet experiments, calculated with CQL3D/KPRAD model Phys. Plasmas 7 4590-99

[50] Smith H, Helander P, Eriksson L-G and Fülöp T 2005 Runaway electron generation in a cooling plasma Phys. Plasmas $\mathbf{1 2}$

[51] Taylor P L et al 1999 Disruption mitigation in DIII-D Phys. Plasmas 6 1872-9 